\title{
The Effect of Selenium Supplementation on Clinical Outcomes, Metabolic Profiles, and Pulsatility Index of the Uterine Artery in High-Risk Mothers in Terms of Preeclampsia Screening with Quadruple Test: a Randomized, Double-Blind, Placebo-Controlled Clinical Trial
}

\section{Selenium and preeclampsia}

\author{
Elahe Mesdaghinia ${ }^{1} \cdot$ Farah Shahin $^{1} \cdot$ Amir Ghaderi $^{2} \cdot$ Daryoush Shahin $^{3} \cdot$ Mohammad Shariat $^{4}$. \\ Hamidreza Banafshe ${ }^{5}$
}

Received: 20 December 2021 / Accepted: 22 February 2022 / Published online: 28 February 2022

(c) The Author(s), under exclusive licence to Springer Science+Business Media, LLC, part of Springer Nature 2022

\begin{abstract}
Data on the effects of selenium (Se) supplementation on clinical outcomes, metabolic profiles, and pulsatility index (PI) in high-risk mothers in terms of preeclampsia (PE) screening with quadruple tests are scarce. This study evaluated the effects of Se supplementation on clinical outcomes, metabolic profiles, and uterine artery PI on Doppler ultrasound in high-risk mothers in terms of PE screening with quad marker. The current randomized, double-blind, placebo-controlled trial was conducted among 60 high-risk pregnant women screening for PE with quad tests. Participants were randomly allocated into two groups (30 participants each group), received either $200 \mu \mathrm{g}$ /day Se supplements (as Se amino acid chelate) or placebo from 16 to 18 weeks of pregnancy for 12 weeks. Clinical outcomes, metabolic profiles, and uterine artery PI were assessed at baseline and at the end of trial. Se supplementation resulted in a significant elevation in serum Se levels ( $\beta 22.25 \mu \mathrm{g} / \mathrm{dl} ; 95 \%$ CI, 18.3, 26.1; $P<0.001)$ compared with the placebo. Also, Se supplementation resulted in a significant elevation in total antioxidant capacity ( $\beta 82.88 \mathrm{mmol} / \mathrm{L} ; 95 \% \mathrm{CI}, 3.03,162.73 ; P=0.04)$, and total glutathione $(\beta 71.35 \mu \mathrm{mol} / \mathrm{L} ; 95 \% \mathrm{CI}, 5.76$, $136.94 ; P=0.03)$, and a significant reduction in high-sensitivity $\mathrm{C}$-reactive protein levels $(\beta-1.52 ; 95 \% \mathrm{CI},-2.91,-0.14$; $P=0.03$ ) compared with the placebo. Additionally, Se supplementation significantly decreased PI of the uterine artery in Doppler ultrasound $(\beta-0.09 ; 95 \% \mathrm{CI},-0.14,-0.04 ; P=0.04)$, and a significant improvement in depression $(\beta-5.63$; 95\% CI $,-6.97,-4.28 ; P<0.001)$, anxiety $(\beta-1.99 ; 95 \% \mathrm{CI},-2.56,-1.42 ; P<0.001)$, and sleep quality $(\beta-1.97 ; 95 \%$ CI, $-2.47,-1.46 ; P<0.001$ ). Se supplementation for 12 weeks in high-risk pregnant women in terms of PE screening with quad marker had beneficial effects on serum Se level, some metabolic profiles, uterine artery PI, and mental health. IRCT Registration: htpp://www.irct.ir; identifier IRCT20200608047701N1.
\end{abstract}

Keywords Selenium · Preeclampsia $\cdot$ Uterine artery pulsatility index $\cdot$ Doppler ultrasound $\cdot$ Quad test $\cdot$ Metabolic profiles

Farah Shahin

Farahshahin660@yahoo.com

1 Department of Gynecology and Obstetrics, School of Medicine, Kashan University of Medical Sciences, Kashan, Islamic Republic of Iran

2 Department of Addiction Studies, School of Medicine AND Clinical Research Development Unit,
Matini/Kargarnejad Hospital, Kashan University of Medical Sciences, Kashan, Iran

3 Amelia Heart AND Vascular Center, Springfield, VA, USA

4 Department of Pathology and Histology, Laboratory of Dr. Shariat, Kashan, Iran

5 Department of Pharmacology, School of Medicine, Kashan University of Medical Sciences, Kashan, Iran 


\section{Introduction}

Preeclampsia (PE) and related hypertensive disorders of pregnancy complicate $2-5$ percentages of all pregnancies in developed countries and are major contributors to maternal and neonatal morbidities and mortalities [1, 2]. PE and eclampsia rank second or third in all worldwide ranking of maternal death causes. In Iran, PE is reported to be involved in $12 \%$ of pretermed neonate deliveries and $18.2 \%$ of maternal deaths. Also, the prevalence of the disease is not equal among different ethnic groups [3-5]. $\mathrm{PE}$ is a major disease of human pregnancy, marked by hypertension and proteinuria, appearing during the second or third trimester of gestation [6,7]. There are risk factors and clinical factors that significantly increase PE risk, including the extremes of maternal age, chronic hypertension, family predisposition for PE and eclampsia, polycystic ovarian syndrome, nulliparity, new male partner, multiple gestations, obesity, sleep disordered breathing, preexisting diabetes, and various infections such as periodontal disease, urinary tract infections, renal disease, and helicobacter pylori. Also, in terms of obstetric history, vaginal bleeding for at least 5 days during pregnancy increases PE risk $[8,9]$. Despite several studies on PE, its exact etiology remains poorly understood. However, it is known that placental changes occur early in pregnancy, associated with an imbalance between the antioxidant defense system, and the generation of reactive oxygen species (ROS) characterizing oxidative stress. Also, there is a generalized inflammatory process, as well as the presence of progressive vascular endothelial damage, which culminates in placental dysfunction $[10,11]$. However, the placenta is naturally protected by some antioxidant defenses. These antioxidant enzymes' defense systems help to prevent the placenta from undue harm. This is the basis of the involvement of micronutrients and other antioxidants in the etiopathogenesis of PE [12]. In addition, hypertension, dyslipidemia, insulin resistance, endothelial dysfunction, dysfunction hemoglobin, platelets, hepatic transaminases, protein/creatinine ratio, and uric acid, and vascular impairment have all been observed in PE [13]. Also, PE has negative effects on the psycho-emotional state (depression and anxiety), sleep patterns, and psychopathological symptoms $[14,15]$.

$\mathrm{Se}$ is an essential micronutrient, which plays an important role in preserving many natural body functions. The biological function of $\mathrm{Se}$ is related to its incorporation through selenocysteine (SeCys) into the structure of proteins important for metabolism [16-18]. Se is present in both inorganic and organic forms. The organic form is the Se contained in amino acids termed as selenomethionine (SeMet) and SeCys, while the inorganic forms (e.g., selenate, selenite, selenide, and elemental Se). The inorganic form of Se is a major source of dietary Se in the human population. Animals that are raised on Secontaining soil are a rich source of selenoproteins (SePs) $[16,19]$. SePs play various functions in the human body, including cell signaling and redox reactions, selenium transport (selenoprotein $\mathrm{P}$ ), and activation and proliferation of immune cells (selenoprotein K) [17, 20]. It constitutes an integral part of selenoproteins and some antioxidant enzymes such as thioredoxin reductase (TRxR), glutathione peroxidase (GPx), and iodothyronine deiodinase (DIO), which protect cells from the damaging effects of free radicals produced during oxidation [16]. Also, Se is a component of other enzymes, particularly DIO which catalyzes the deionization of thyroxine (T4) to triiodothyronine (T3). Deiodinases play a key role in the regulation of thyroid hormones. They are responsible for the control of proper growth, development, and cell metabolism [16]. Additionally, Se plays a key role in the immune system regulation. The element stimulates the immune system to enhance the production of antibodies ( $\mathrm{IgM}$ and $\mathrm{IgG}$ ) and causes enhanced activity of macrophages and $\mathrm{T}$ cells [21]. Also, the protective effects of Se in the etiology of cancer result from its effect on cell membranes protecting against oxidative stress, and the stabilizing effect on DNA and increase of cellular immune response. It has been indicated that Se inhibits tumor cell proliferation via the effect exerted on Bcl-2 apoptosis suppressor gene and the expression of p53 tumor suppressor gene [18, 22]. Protein hydrophobic interactions induced by a variety of nutritional and environmental factors can provide rational explanation for a number of pathologic conditions. The deficiency of Se leads to elevated pathological changes, inflammation, and oxidative stress [23, 24]. Several studies have assessed the relationship between the level of body Se and the incidence, severity, and mortality of different disease such as cancer, COVID-19, Alzheimer, respiratory diseases, hepatic diseases, polycystic ovary syndrome (PCOS), cardiovascular disorders, neurologic, and other degenerative diseases [25-29].

Previous studies have reported controversial results between Se levels and PE, recurrent abortions, and intrauterine growth restriction (IUGR) [30, 31]. Se is a component of some vital enzymes that play roles in the antioxidant activities, so if serum values of these enzymes are low, it can result in numerous pathways that can lead to PE [32]. Also, with increased understanding of the pathogenesis of PE, clinical prediction models of the disease have gradually diversified. Doppler has more recently shown some promise [33]. First and second trimester uterine artery Doppler assessments have high predictive value for clinical outcome (prediction of PE and IUGR) [34]. Uterine artery PI has been used as a marker of PE and 
fetal growth restriction (FGR), in the presence of which PI increases due to the elevation in uterine artery impedance [35]. Combination of the clinical, biochemical, and biophysical markers is likely to increase the predictive power of screening examinations [36]. Currently, the implementation of serum biomarker screening quad test (AFP, alpha-fetoprotein; BHCG, beta-human gonadotropin; uE3, unconjugated estriol; and IHA, inhibin-A) for fetal aneuploidy has commenced worldwide, and many studies have shown that additional information derived from such screenings, other than aneuploidy risk estimation, can also be used to identify the risk of adverse obstetric outcomes, such as fetal growth restriction, PE, and preterm birth [37, 38].

The hypothesis of this clinical trial is that clinical outcomes, metabolic profiles, PI of uterine artery, and mental health might improve among high-risk mothers in terms of PE screening with quadruple tests. A literature review revealed limited information about the effects of Se on clinical profiles, mental health, PI and metabolic biomarkers in high-risk mothers screening with quadruple tests. Therefore, in this clinical trial, we aimed to evaluate the effects of Se in pregnant women at the risk of PE screened with quad test.

\section{Methods}

\section{Trial Design and Participants}

This research was a prospective randomized double-blind placebo-controlled clinical trial. The current study was done among 60 high-risk pregnant women in terms of PE that were screened by quad test, aged 18-40 years who referred to Shahid Beheshti Hospital, Kashan, Iran, between May 2020 and December 2020. Participants who consumed Se supplements during the past 12 weeks and pregnant women with diabetes mellitus, thyroid disorders, hypertension, chronic proteinuria, abnormal scan anomaly, multiplication, AIDS, hepatitis B or hepatitis C, intolerance to yeasts containing selenium supplements, and bone and chromosomal abnormalities were excluded.

\section{Ethics Statements}

This clinical trial was done according to the guidelines laid down in the Declaration of Helsinki. This study was approved by the ethics committee of Kashan University of Medical Sciences (IR.KAUMS.MEDNT.REC.1399.008) and registered on the Iranian Registry of Clinical Trials website (http://www.irct.ir: IRCT20200608047701N1). All subjects provided informed written consent before recruitment.

\section{Clinical Trial Procedures}

Randomization was done using computer-generated random numbers by a trained staff at the obstetrics and gynecology clinic. Randomization and allocation were concealed to the researchers and participants until the final analyses were completed. Another person at the clinic, who was not involved in the trial and not aware of random sequences, assigned the participants to the numbered bottles of supplement. The treatment group $(n=30)$ received $200 \mu \mathrm{g} / \mathrm{day} \mathrm{Se}$ as Se amino acid chelate (Alfa Vitamins, Florida, USA) from 16 to 18 weeks of gestation for 12 weeks with meals. Placebo group $(n=30)$ received capsules in size, shape, color, packaging, taste, and smell as Se. Participants were asked not to take other Se supplements during the 12-week intervention, however maintaining their regular diet and physical activity throughout the intervention period. Adherence to supplements and placebos was determined by counting the tablet containers. To increase compliance, all participants received brief daily cell phone reminders to take the Se and Placebo.

\section{Assessment of Anthropometric Measures}

Weight and height of participants were determined in an overnight fasting status using a standard scale (Seca, Hamburg, Germany) at the baseline and after the 3-month intervention. Body mass index (BMI) was calculated as weight in kilograms divided by height in meters squared.

\section{Assessment of Outcomes}

The primary outcome measurement was high-sensitivity C-reactive protein (hs-CRP), and the secondary outcome measurements were clinical outcomes, other metabolic profiles, and uterine artery PI.

\section{Biochemical Measures}

At first and after the end of the clinical trial, $10 \mathrm{~mL}$ of blood samples and a urine sample were taken from each subject at the Kashan Reference Laboratory in an early morning after an overnight fast (Kashan, Iran). Blood was collected in two separate tubes: (A) one without EDTA to separate the serum, in order to determine serum Se, aspartate aminotransferase (AST), alanine aminotransferase (ALT), lactate dehydrogenase (LDH), uric acid, prolactin, and hs-CRP concentrations and (B) another one containing EDTA to examine plasma nitric oxide (NO), total antioxidant capacity (TAC), total glutathione (GSH), and malondialdehyde (MDA). Also, protein/creatinine ratio was measured in urine samples. Blood samples were immediately centrifuged (Hettich D-78532, Tuttlingen, Germany) 
at $3500 \mathrm{rpm}$ for $10 \mathrm{~min}$ to separate serum and plasma. Then, the samples were stored at $-80{ }^{\circ} \mathrm{C}$ before analysis at the KUMS reference laboratory. Hemoglobin, hepatic transaminases, protein/creatinine ratio, and uric acid were measured on the day of blood and urine sampling with enzymatic kits (Pars Azmun, Tehran, Iran) with intra-assay coefficient of variations (CV s) below 5\%. Platelet counts in venous whole blood samples were determined with Sysmex KX-21 Haematology analyzer (Sysmex Corporation, Japan). Also, the samples were analyzed to determine the serum Se levels, using atomic absorption spectrometry method (A Perkin-Elmer) [39]. Plasma TAC concentrations were measured using the method of ferric reduction antioxidant power developed by Benzie and Strain [40]. Plasma GSH using the method of Tipple et al. [41], and MDA concentrations by the thiobarbituric acid reactive substances spectrophotometric test were determined with inter- and intra-assay CVs below 5\% [42]. A commercial ELISA kit (LDN, Nordhorn, Germany) with inter- and intra-assay CVs below 7\% determined serum hs-CRP levels. The plasma NO levels were determined using Griess method [43]. Measurements of metabolic biomarkers were performed in a blinded fashion, in duplicate (preand post-intervention) after the intervention, in the same analytical run, and in random order to reduce systematic error and inter-assay variability.

\section{Clinical Measures}

Mean validated automated devices (3BTO-A2, Micro life, Taipei, Taiwan) measured arterial pressure. Women were in the sitting position with their arms supported at the level of the heart, and a small $(22 \mathrm{~cm})$, normal $(22-32 \mathrm{~cm})$, or large $(33-42 \mathrm{~cm})$ adult cuff was used, depending on the mid-arm circumference. After resting for $5 \mathrm{~min}$, two recordings of blood pressure were made in both arms simultaneously. We calculated the final MAP as the average of all four measurements [44]. In the present study, Beck's Depression (BDI), Anxiety (BAI), and the Pittsburgh Sleep Quality Index (PSQI) inventories were used to assess level of depression, anxiety, and sleep quality, respectively [45-47]. BDI is a 21-question and BAI is a 20-question inventory, where each question scored between 0 and 4 , and higher scores indicate the higher levels of depression and anxiety, respectively. The Persian versions of both inventories were validated in the previous studies $[48,49]$. The PSQI includes a scoring key for calculating a patient's seven sub-scores, each of which can range from 0 to 3 . The sub-scores are tallied, yielding a "global" score that can range from 0 to 21 . A global score of 5 or more indicates poor sleep quality; the higher the score, the worse the quality [47].

\section{Radiology Measures}

Trans-abdominal color Doppler ultrasound was performed before and after the intervention to evaluate the left and right uterine artery PI, and the average value was recorded. This examination was performed using trans-abdominal ultrasound with color-flow mapping. When three similar consecutive wave forms were provided, the PI was measured on each side.

\section{Sample Size}

We did not detect a similar trial about the effects of Se supplementation on uterine artery PI, clinical signs, and metabolic profiles in high-risk mothers in terms of PE screening with quad marker for determining the sample size based on primary outcome. Hence, the sample size was calculated based on the effects of Se supplementation on hs-CRP status in pregnant women at risk for intrauterine growth restriction (IUGR). Type one $(\alpha)$ and type two $(\beta)$ errors were defined as 0.05 and 0.20 (power $=80 \%$ ), respectively. Based on a previous study [50], we used a standard deviation (SD) of 1.8 and 1 for Se and placebo groups, and a difference in mean (d) of 1.2, considering hs-CRP as the key variable. We needed 24 patients in each group. Assuming a dropout of 6 persons per group, the sample size was considered to be 30 patients in each group.

\section{Statistical Analyses}

The Kolmogorov-Smirnov test was used to determine the normality of data. To detect the differences in anthropometric parameters among two groups, we used the independentsamples $t$-test. Multiple linear regression models were used to assess treatment effects on study outcomes after adjusting for baseline concentration of variables. The effect sizes were demonstrated as the mean differences with $95 \%$ confidence intervals. $P$ values $<0.05$ were considered statistically significant. Statistical analyses were done using the Statistical Package for Social Science version 18 (SPSS Inc., Chicago, Illinois, USA).

\section{Results}

Six subjects in the supplements group and four subjects in the placebo group dropped out for personal reasons (Fig. 1). Finally, 50 patients (selenium $(n=24)$ and placebo $(n=26)$ ) completed the trial. No difference in mean age and anthropometric parameters was seen between the two groups (Table 1). Overall, the compliance rate was high, such that 
Fig. 1 Summary of patient flow diagram

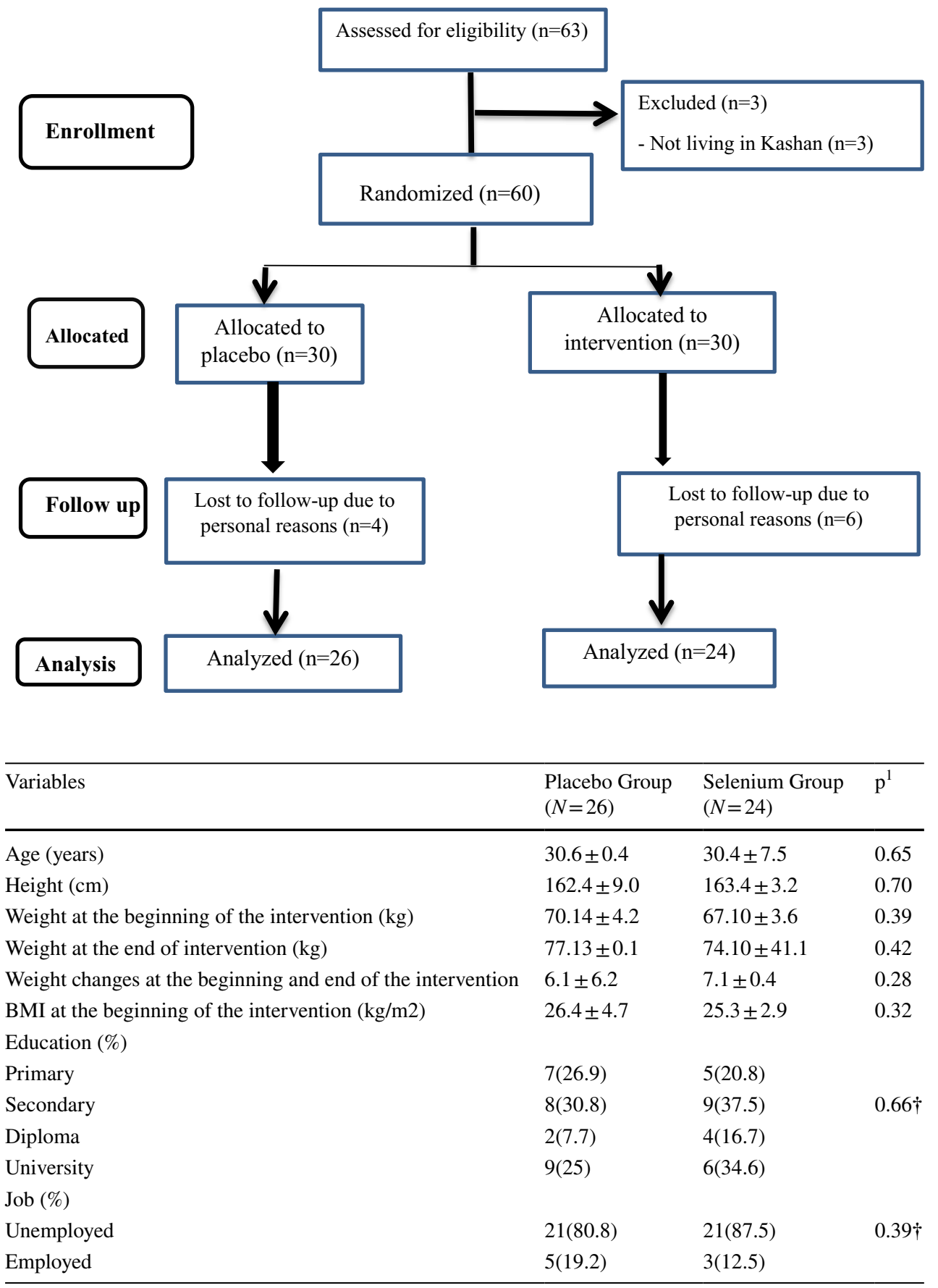

${ }^{1}$ Data are mean \pm SDs and percentage

${ }^{2}$ Obtained from independent $t$-test

${ }^{\dagger}$ Obtained from Pearson chi-square test more than $90 \%$ of capsules were consumed throughout the study in both groups. No side effects were reported following the intake of Se in patients with PE throughout the study.

Se supplementation resulted in a significant improvement in depression scores $(\beta-5.63$; 95\% CI, $-6.97,-4.28$; $P<0.001)$, anxiety scores $(\beta-1.99 ; 95 \% \mathrm{CI},-2.56,-1.42$; $P<0.001)$, and sleep quality scores $(\beta-1.97 ; 95 \%$
CI, $-2.47,-1.46 ; P<0.001)$ compared with the placebo (Table 2). Compared with the placebo, Se supplementation resulted in a significant elevation in TAC $(\beta 82.88 \mathrm{mmol} / \mathrm{L}$; 95\% CI, 3.03, 162.73; $P=0.04)$, and GSH $(\beta 71.35 \mu \mathrm{mol} / \mathrm{L}$; 95\% CI, 5.76, 136.94; $P=0.03$ ), and a significant reduction in hs-CRP levels $(\beta-1.52 \mathrm{mg} / \mathrm{L}$; 95\% CI, $-2.91,-0.14$; $P=0.03$ ). Additionally, Se supplementation significantly 
Table 2 Clinical parameters, metabolic profile, uterine artery PI, and mental health scale at baseline and after 12 weeks of the intervention in high-risk pregnant women in terms of PE screening with quad marker

\begin{tabular}{|c|c|c|c|c|c|c|}
\hline \multirow[t]{2}{*}{ Variables } & \multicolumn{2}{|l|}{$\begin{array}{l}\text { Placebo group } \\
(n=26)\end{array}$} & \multicolumn{2}{|l|}{$\begin{array}{l}\text { Selenium group } \\
(\mathrm{n}=24)\end{array}$} & \multicolumn{2}{|c|}{$\begin{array}{l}\text { Difference in outcome measures } \\
\text { between selenium and placebo treat- } \\
\text { ment groups }^{1}\end{array}$} \\
\hline & Baseline & Week 12 & Baseline & Week 12 & $\beta(95 \% \mathrm{CI})$ & $\mathrm{P}^{2}$ \\
\hline Selenium) $\mu \mathrm{g} / \mathrm{l})$ & $62.1 \pm 12.4$ & $65.7 \pm 12.2$ & $59.8 \pm 8.9$ & $85.6 \pm 12.5$ & $22.25(18.3,26.1)$ & $<0.001$ \\
\hline Blood pressure (mmhg) & $11.0 \pm 9.4$ & $11.1 \pm 8.8$ & $10.7 \pm 8.4$ & $11.2 \pm 7.8$ & $3.62(-0.15,7.40)$ & 0.06 \\
\hline Hemoglobin $(\mathrm{g} / \mathrm{dl})$ & $12.1 \pm 0.7$ & $12.0 \pm 0.7$ & $12.4 \pm 0.8$ & $12.2 \pm 0.6$ & $-0.05(-0.29,0.18)$ & 0.64 \\
\hline Platelets $(\times 1000 / \mu)$ & $184,000 \pm 46,138$ & $181,310 \pm 41,153$ & $208,210 \pm 66,153$ & $207,670 \pm 71,047$ & $40,950.9(-9990.3,18,182.2)$ & 0.56 \\
\hline AST (U/l) & $26.1 \pm 10.4$ & $25.9 \pm 9.5$ & $24.6 \pm 8.4$ & $26.9 \pm 8.5$ & $2.35(-0.65,5.35)$ & 0.12 \\
\hline $\operatorname{ALT}(\mathrm{U} / \mathrm{l})$ & $26.7 \pm 9.6$ & $28.2 \pm 9.0$ & $25.0 \pm 8.8$ & $27.6 \pm 8.8$ & $0.69(-2.48,3.86)$ & 0.66 \\
\hline LDH (IU/l) & $265.5 \pm 45.5$ & $264.3 \pm 53.1$ & $266.5 \pm 48.3$ & $271.6 \pm 45.0$ & $6.63(-14.0,27.34)$ & 0.52 \\
\hline Creatinine (mg/dl) & $0.7 \pm 0.05$ & $0.7 \pm 0.04$ & $0.7 \pm 0.06$ & $0.7 \pm 0.05$ & $0.003(-0.02,0.02)$ & 0.78 \\
\hline Uric acid (mg/dl) & $3.8 \pm 0.8$ & $4.0 \pm 0.6$ & $3.8 \pm 0.6$ & $4.0 \pm 0.7$ & $-0.08(-0.34,0.17)$ & 0.52 \\
\hline Urine $\mathrm{Pr} / \mathrm{Cr}$ ratio & $0.02 \pm 0.01$ & $0.02 \pm 0.01$ & $0.01 \pm 0.01$ & $0.02 \pm 0.009$ & $-0.005(-0.01,-0.002)$ & 0.13 \\
\hline hs-CRP (mg/L) & $7.5 \pm 5.1$ & $7.7 \pm 4.5$ & $9.0 \pm 4.8$ & $7.1 \pm 2.5$ & $-1.52(-2.91,-0.14)$ & 0.03 \\
\hline $\mathrm{NO}(\mu \mathrm{mol} / \mathrm{L})$ & $65.2 \pm 11.4$ & $69.0 \pm 14.1$ & $64.0 \pm 7.7$ & $72.2 \pm 11.2$ & $4.54(-2.24,11.34)$ & 0.18 \\
\hline $\mathrm{TAC}(\mathrm{mmol} / \mathrm{L})$ & $715.7 \pm 141.0$ & $730.5 \pm 156.2$ & $727.9 \pm 144.8$ & $827.1 \pm 236.1$ & $82.88(3.03,162.73)$ & 0.04 \\
\hline $\mathrm{GSH}(\mu \mathrm{mol} / \mathrm{L})$ & $729.2 \pm 183.7$ & $744.5 \pm 183.6$ & $742.9 \pm 210.9$ & $833.6 \pm 246.8$ & $71.35(5.76,136.94)$ & 0.03 \\
\hline $\mathrm{MDA}(\mu \mathrm{mol} / \mathrm{L})$ & $4.8 \pm 1.7$ & $5.2 \pm 1.7$ & $4.9 \pm 1.4$ & $4.7 \pm 1.3$ & $-0.57(-1.40,0.25)$ & 0.17 \\
\hline UAPI & $1.31 \pm 0.26$ & $0.88 \pm 0.13$ & $1.34 \pm 0.27$ & $0.8 \pm 0.09$ & $-0.09(-0.14,-0.04)$ & 0.04 \\
\hline Depression score & $10.07 \pm 5.13$ & $10 \pm 5.15$ & $10.91 \pm 3.8$ & $4.83 \pm 1.73$ & $-5.63(-6.97,-4.28)$ & $<0.001$ \\
\hline Anxiety score & $8.11 \pm 4.05$ & $7.23 \pm 3.46$ & $5.79 \pm 2.55$ & $3.33 \pm 2.14$ & $-1.99(-2.56,-1.42)$ & $<0.001$ \\
\hline Sleep quality & $6.15 \pm 3.68$ & $5.53 \pm 3.38$ & $5.58 \pm 1.97$ & $3.04 \pm 1.33$ & $-1.97(-2.47,-1.46)$ & $<0.001$ \\
\hline
\end{tabular}

Data are mean \pm SDs

1 "Outcome measures" refers to the change in values of measures of interest between baseline and week 12 . $\beta$ (difference in the mean outcomes measures between treatment groups $($ Se group $=1$ and placebo group $=0)$ )

${ }^{2}$ Obtained from multiple regression model (adjusted for baseline values of each clinical and biochemical variables)

AST aspartate aminotransferase; $A L T$ alanine aminotransferase; $L D H$ lactate dehydrogenase; urine (Pr/Cr) ratio, urine (protein/creatinine) ratio; $h s-C R P$ high-sensitivity C-reactive protein; $N O$ nitric oxide; $T A C$ total antioxidant capacity; $G S H$ total glutathione; $M D A$ malondialdehyde; $U A P I$ uterine artery pulsatility index

increased serum Se levels ( $\beta 22.25 \mathrm{mg} / \mathrm{dL}$; 95\% CI, 18.3, $26.1 ; P<0.001)$, and a significant decrease in UAPI $(\beta-0.09$; $95 \% \mathrm{CI},-0.14,-0.04 ; P=0.04)$ compared with the placebo. Se supplementation did not affect other metabolic profiles.

\section{Discussion}

In the current study, we investigated the effects of supplementation of Se on clinical symptoms, metabolic status, and uterine artery PI in high-risk pregnant women in terms of PE screening with quad marker. We found that taking Se supplements for 12 weeks by high-risk pregnant women in terms of PE screening with quad marker improved the depression, anxiety, sleep quality scores, UAPI, Se, hs-CRP, TAC, and GSH levels, but did not affect blood pressure, hemoglobin, platelets, AST, ALT, LDH, creatinine, uric acid, protein/ creatinine ratio, MDA, and NO levels. Based on these findings, supplementation of Se may be an appropriate adjunct therapy for high-risk pregnant women in terms of PE screening with quad marker. To our knowledge, this evidence is the first study to evaluate the effects of Se on clinical outcomes, metabolic biomarkers, and uterine artery PI in high-risk pregnant women in terms of PE screening with quad marker. Previous evidence has demonstrated that a small increase in Se intake in pregnant women with inadequate Se status could protect them against PE [51]. In the current study, no side effects were seen following supplementation with $\mathrm{Se}$ in high-risk pregnant women in terms of PE screening with quad marker throughout the study. It must be considered that mean dietary plus supplemental Se administration in our study patients was lower than upper limits $(400 \mu \mathrm{g})$. Se is an element with a very narrow quantitative range of levels between deficiency, physiological responses, and toxic dose [16]. Excess of Se in the diet causes chronic food poisoning signs (e.g., nausea, vomiting, and diarrhea). Acute exposure to high amounts of Se leads to a general weakness and neurological disorders [52]. Also, chronic toxicity by an 
excess of Se leads to selenosis signs, which is manifested by changes and fragility of fingernails, hair loss, infertility, skin rash, gastrointestinal disorders, unpleasant odor in exhaled air, and nervous system disorders [16, 53]. However, data on the toxic effects of Se supplementation on health situation are conflicting. In the study conducted by Burk et al. [54], it indicated that administration of moderate $200 \mu \mathrm{g} /$ day to large dose $600 \mu \mathrm{g} /$ day Se was safe in volunteers ages $\geq 18$ years for 16 weeks. On the other hand, gastrointestinal signs, hair loss, and memory disorder were reported as the adverse effects of Se intake [55]. However, further evidences are required about potential teratogenicity/toxicity of long-term increased Se intake in high-risk pregnant women in terms of PE screening. Although the beneficial effects of Se $(100 \mu \mathrm{g} / \mathrm{day})$ from the first trimester of their pregnancy until delivery for a period of approximately 6 months were reported [56], we believe that further studies are required to confirm our results.

\section{Effects on Clinical Outcomes and Uterine Artery PI}

Previous studies have shown that Se administration has the potential to decrease the risk of $\mathrm{PE}$ in pregnant women with low Se levels. Se is known as an essential trace mineral that has many important functions at the concentration of the cell and organism in human and animal health, and so it is relevant to various pathophysiological conditions [57]. We found that supplementation of Se after 12 weeks to high-risk pregnant women in terms of PE screening with quad marker improved the Se levels, and UAPI, but it did not affect the total clinical outcomes, compared with the placebo. Data documenting the effects of supplementation of Se on clinical outcomes and uterine artery PI in high-risk pregnant women in terms of PE screening with quad marker are limited. In a study by Gomez et al. [58], successive changes in uterine artery blood flow patterns between the first and second trimesters of pregnancy and their association with risk of hypertensive disorders and fetal IUGR were investigated. The results showed that compared to normal pregnancies, complication pregnancies have a higher prevalence of bilateral notch and uterine artery PI in each of the first and second trimesters. Pregnant women with sustained abnormal PI are at greater risk for adverse perinatal outcomes. In addition, in a study conducted by Mesdaghinia et al. [50], it was observed that supplementation with Se for 10 weeks led to a higher percentage of women in the Se group had PI of $<1.45$ than in the placebo group.

\section{Effects on Metabolic Biomarkers}

There are placental changes in PE, linked to an imbalance between the production of ROS and the antioxidant defense system, characterizing the placental oxidative stress that leads to an increase in the production of pro-inflammatory cytokines [11]. Our study indicated that taking Se supplements for 12 weeks in high-risk pregnant women in terms of PE screening with quad marker resulted in a significant rise in plasma GSH and TAC, and a significant reduction in serum hs-CRP levels compared with the placebo, but unchanged plasma MDA and NO levels. As there is evidence that Se supplementation may have antioxidant and anti-inflammatory effects $[59,60]$. In a study done by Kamali et al. [61], it was seen that the intake of $200 \mu \mathrm{g} /$ day Se supplements for 4 weeks to coronary artery bypass grafting (CABG) surgery had beneficial effects on hs-CRP, GSH, and MDA levels, but did not affect other metabolic profiles. Also, total antioxidant status (TAS), and GSH levels had significant increase following supplementation with Se after 45 days in patients with refractory epilepsy, and epilepsy [62]. In addition, results of a meta-analysis in randomized controlled trials indicated a reduction in serum CRP and increased the glutathione peroxidase level in patients with coronary heart disease supplemented with Se [63]. However, in a study conducted by Savory et al. [64], Se supplementation at a dosage of $200 \mu \mathrm{g}$ daily for 3 weeks among overweight adults did not alter GSH, and TAS levels. Se appeared to provide a protective effect against oxidative stress, and $\mathrm{Ca}(2+)$ entry through modulation of neutrophil transient receptor potential vanilloid 1 (TRPV1) calcium channels in patients with PCOS [65]. Also, Se intake may decrease inflammation and oxidative stress through increasing selenoprotein biosynthesis, inhibiting production of ROS/RNS, and the inhibition of nuclear factor kappa light chain-enhancer of activated B cells (NF-kappa B) by modulating selenoprotein gene expressions [66-68].

\section{Effects on Mental Health Parameters}

Se deficiency occurred significantly more among the PE pregnant women compared to the normotensive controls [32], and low Se status might experience relatively depressed moods, and support the idea that Se plays a special role in the brain [69]. PE is a pregnancy-associated condition with complex disease mechanisms, and a risk factor for various long-term health outcomes for the infant and mother [70]. PE women most often experience complications of mood disorders in comparison with females with uncomplicated pregnancy [71]. Mental health disorder (depression, anxiety, and sleep) are highly prevalent in high-risk pregnancy such as PE $[72,73]$. In our study, a 12-week Se supplementation by high-risk pregnant women in terms of PE screening with quad marker reduced depression, anxiety, and sleep quality scores compared with the placebo. In a study by Mokhber et al. [74], it showed that supplementation with $100 \mu \mathrm{g} / \mathrm{day}$ selenium during pregnancy might be an effective approach for the prevention of postpartum depression level in Iranian 
women. In addition, Se therapy ( $200 \mu \mathrm{g} /$ day) to HIV/drug users led to a significant improvement in decrease anxiety but did not affect depression or distress [75]. Also, current data supports the beneficial effect of Se on central nervous system, depression, and sleep disorders [76]. However, Hawkes and Hornbostel [69] reported that selenium supplementation did not promote improvements in mood in persons eating a typical US diet. While the neuroprotective effect of Se has yet to be definitively characterized, the protective role of Se in psychological disorders could be related to its important action in antioxidant defense [77]. Additionally, Se has been shown to influence health status through its important role in immune function and neurotransmitter turnover rate $[78,79]$. Also, Se intake may affect mental health parameters through modulating thyroid function, which in turn play a role in the reduction of depression score [80].

\section{Limitations}

The current study had few limitations. Firstly, sample size in our study was small. Future studies with longer duration and higher participant are needed to confirm the validity of our finding. In addition, we did not evaluate dietary intakes of study patients; however, we requested patients not to change their regular dietary intakes and physical activity. Furthermore, additional future studies are needed to examine the effect of Se supplementation on gene expression involved in metabolic profiles, and inflammation factors, as well as on Se-dependent antioxidant enzymes including thioredoxin reductase and GPx isoforms. Unfortunately, because of limited funding, we did not examine the effects of Se supplementation on these measures. In addition, the beneficial effects of Se supplementation on lipid profiles and glucose homeostasis would be of interest, and it is suggested for a future study.

\section{Conclusion}

Overall, our study demonstrated that Se supplementation for 12 weeks in high-risk pregnant women in terms of PE screening with quad marker resulted in a significant improvement of depression, anxiety, sleep quality scores, UAPI, Se, GSH, TAC, and hs-CRP levels but did not affect other clinical symptoms and metabolic profiles. This suggests that Se supplementation for 12 weeks at dosage $200 \mu \mathrm{g} /$ day might confer advantageous therapeutic effects for high-risk pregnant women in terms of PE screening with quad marker. Further evidence is required in other diseases and for longer periods to determine the safety of selenium supplement.
Abbreviations PE: Preeclampsia; Se: Selenium; GSH: Total glutathione; hs-CRP: High-sensitivity C-reactive protein; MDA: Malondialdehyde; NO: Nitric oxide; TAC: Total antioxidant capacity; UAPI: Uterine artery pulsatility index

Acknowledgements This study was the thesis on resident of Obstetrics and Gynecology (Farah Shahin). It has been registered with the IRCT20200608047701N1 registration code in the Iranian Center for Clinical Trials, and was supported by a grant from the Vice-chancellor for Research, KAUMS, Iran (99012). We are thankful of all subjects who participated in this project.

Author Contributions FSH, EM, AG, DSH, MSH, and HB contributed in conception, design, statistical analysis, and drafting of the manuscript. All authors approved the final version for submission. FSH supervised the study.

Funding The research grant provided by Research Deputy of Kashan University of Medical Sciences (KAUMS/99012).

Availability of data and materials The datasets generated and/or analyzed during the current study are not publicly available because the intellectual property is owned by the funding body. They may be available from the corresponding author on reasonable request containing the approval from the associated funding body.

\section{Declarations}

Ethics Approval and Consent to Participate At the beginning of the questionnaire distribution session, the purpose of the study was explained for the participants and they were assured about the anonymity and confidentiality of their responses. All participants gave their signed written informed consent letters. The study protocol was approved by the Ethics Committee of Kashan University of Medical Sciences "approval no; IR.KAUMS.MEDNT.REC.1399.008". All procedures performed in studies involving human participants were in accordance with the ethical standards of the institutional and national research committee and with the 1964 Helsinki declaration and its later amendments.

Consent for Publication Not applicable.

Conflict of Interest The authors declare no competing interests.

\section{References}

1. Duley L (2009) The global impact of pre-eclampsia and eclampsia. Semin Perinatol 33(3):130-137

2. Martinez-Portilla R et al (2021) Incidence of pre-eclampsia and other perinatal complications among pregnant women with congenital heart disease: systematic review and meta-analysis. Ultrasound Obstet Gynecol 58(4):519-528

3. Aali BS, Ghafoorian J, Mohamad-Alizadeh S (2004) Severe preeclampsia and eclampsia in Kerman, Iran: complications and outcomes. Med Sci Monit 10(4): CR163-CR167

4. Mayrink J, Costa ML, Cecatti JG (2018) Preeclampsia in 2018: revisiting concepts, physiopathology, and prediction. Sci World J 6(2018):6268276

5. Tanaka $\mathrm{M}$ et al (2007) Racial disparity in hypertensive disorders of pregnancy in New York State: a 10-year longitudinal population-based study. Am J Public Health 97(1):163-170 
6. Morikawa M et al (2021) Earlier onset of proteinuria or hypertension is a predictor of progression from gestational hypertension or gestational proteinuria to preeclampsia. Sci Rep 11(1):12708

7. Poon LC et al (2020) From first-trimester screening to risk stratification of evolving pre-eclampsia in the second and third trimesters of pregnancy: a comprehensive approach. Ultrasound Obstet Gynecol 55(1):5-12

8. Fox R et al (2019) Preeclampsia: risk factors, diagnosis, management, and the cardiovascular impact on the offspring. J Clin Med $8(10): 1625$

9. Stitterich $\mathrm{N}$ et al (2021) Risk factors for preeclampsia and eclampsia at a main referral maternity hospital in Freetown, Sierra Leone: a case-control study. BMC Pregnancy Childbirth 21(1):413

10. Williamson RD et al (2017) Oxidative stress in pre-eclampsia; have we been looking in the wrong place? Pregnancy Hypertens $8: 1-5$

11. Tenório MB et al (2019) Cross-talk between oxidative stress and inflammation in preeclampsia. Oxid Med Cell Longev 4(2019):8238727

12. Yusuf $\mathrm{H}$ et al (2019) Associations of macro and micronutrients and antioxidants intakes with preeclampsia: A case-control study in Jordanian pregnant women. Nutr Metab Cardiovasc Dis 29(5):458-466

13. Grandi SM et al (2019) Cardiovascular disease-related morbidity and mortality in women with a history of pregnancy complications: systematic review and meta-analysis. Circulation 139(8):1069-1079

14. Caropreso L et al (2020) Preeclampsia as a risk factor for postpartum depression and psychosis: a systematic review and metaanalysis. Arch Womens Ment Health 23(4):493-505

15. Cetin $O$ et al (2017) Investigation of maternal psychopathological symptoms, dream anxiety and insomnia in preeclampsia. J Matern Fetal Neonatal Med 30(20):2510-2515

16. Kieliszek M, Błażejak S (2016) Current knowledge on the importance of selenium in food for living organisms: a review. Molecules 21(5):609

17. Kieliszek M, Bano I, Zare H (2021) A comprehensive review on selenium and its effects on human health and distribution in Middle Eastern Countries. Biol Trace Elem Res 200(3):971-987

18. Kieliszek M, Błażejak S (2013) Selenium: significance, and outlook for supplementation. Nutrition 29(5):713-718

19. Kieliszek M (2019) Selenium-fascinating microelement, properties and sources in food. Molecules 24(7):1298

20. Gladyshev VN et al (2016) Selenoprotein gene nomenclature. J Biol Chem 291(46):24036-24040

21. Ruseva B, Himcheva I, Nankova D (2013) Importance of selenoproteins for the function of the thyroid gland. Medicine 3:60-64

22. Zabłocka K, Biernat J (2010) The influence of selected nutritional components in lung cancer risk - unsaturated fatty acids, isothiocyanates, selenium. Contemporary Oncology/Współczesna Onkologia 14(1):54-58. https://doi.org/10.5114/wo.2010.979

23. Tang $\mathrm{C}$ et al (2020) Selenium deficiency-induced redox imbalance leads to metabolic reprogramming and inflammation in the liver. Redox Biol 36:101519.

24. Kieliszek M, Lipinski B (2018) Pathophysiological significance of protein hydrophobic interactions: An emerging hypothesis. Med Hypotheses 110:15-22

25. Vaz FNC et al (2018) The relationship between copper, iron, and selenium levels and alzheimer disease. Biol Trace Elem Res 181(2):185-191

26. Fakhrolmobasheri $\mathrm{M}$ et al (2021) COVID-19 and selenium deficiency: a systematic review. Biol Trace Elem Res 5:1-12

27. Lin CC et al (2006) Selenium, iron, copper, and zinc levels and copper-to-zinc ratios in serum of patients at different stages of viral hepatic diseases. Biol Trace Elem Res 109(1):15-24
28. Sharma P et al (2022) Assessment of Serum elements concentration and polycystic ovary syndrome (PCOS): systematic review and meta-analysis. Biol Trace Elem Res. https://doi.org/10.1007/ s12011-021-03058-6

29. Navarro-Alarcón M, López-Martínez MC (2000) Essentiality of selenium in the human body: relationship with different diseases. Sci Total Environ 249(1-3):347-371

30. Mistry HD et al (2012) Selenium in reproductive health. Am J Obstet Gynecol 206(1):21-30

31. Zadrożna M et al (2009) Antioxidants activities and concentration of selenium, zinc and copper in preterm and IUGR human placentas. J Trace Elem Med Biol 23(2):144-148

32. Eze SC et al (2020) Serum selenium levels of pre-eclamptic and normal pregnant women in Nigeria: A comparative study. PLoS One 27;15(8):e0238263.

33. Pedroso MA et al (2018) Uterine artery Doppler in screening for preeclampsia and fetal growth restriction. Rev Bras Ginecol Obstet 40(5):287-293

34. Cnossen JS et al (2008) Use of uterine artery Doppler ultrasonography to predict pre-eclampsia and intrauterine growth restriction: a systematic review and bivariable meta-analysis. CMAJ 178(6):701-711

35. Rashid $\mathrm{M}$ et al (2020) Uterine artery Doppler indices as predictive measures for the pre-eclampsia and intrauterine growth restriction. Biological and Clinical Sciences Research Journal 2020(1). https://doi.org/10.54112/bcsrj.v2020i1.23

36. Abdi F et al (2018) A systematic review of first trimester biochemical and molecular predictive tests for preeclampsia. Curr Hypertens Rev 14(1):21-28

37. Boonpiam R et al (2020) Quad test for fetal aneuploidy screening as a predictor of small-for-gestational age fetuses: a populationbased study. BMC Pregnancy Childbirth 20(1):621

38. Yue CY, Zhang CY, Ying CM (2020) Serum markers in quadruple screening associated with adverse pregnancy outcomes: a casecontrol study in China. Clin Chim Acta 511:278-281

39. Welz B, Melcher M, Schlemmer G (1983) Determination of selenium in human blood serum. Comparison of two atomic-absorption spectrometric procedures. Z Anal Chem 316(2):271-276

40. Benzie IF, Strain JJ (1996) The ferric reducing ability of plasma (FRAP) as a measure of "antioxidant power": the FRAP assay. Anal Biochem 239(1):70-76

41. Tipple TE, Rogers LK (2012) Methods for the determination of plasma or tissue glutathione levels. In: Harris C., Hansen J. (eds) Developmental Toxicology. Methods in Molecular Biology (Methods and Protocols), vol 889. Humana Press, Totowa, NJ. https://doi.org/10.1007/978-1-61779-867-2_20

42. Janero DR (1990) Malondialdehyde and thiobarbituric acid-reactivity as diagnostic indices of lipid peroxidation and peroxidative tissue injury. Free Radic Biol Med 9(6):515-540

43. Tatsch E et al (2011) A simple and inexpensive automated technique for measurement of serum nitrite/nitrate. Clin Biochem 44(4):348-350

44. Poon $\mathrm{L}$ et al (2012) Protocol for measurement of mean arterial pressure at 11-13 weeks' gestation. Fetal Diagn Ther 31(1):42-48

45. Beck AT, Steer RA, Carbin MG (1988) Psychometric properties of the Beck Depression Inventory: twenty-five years of evaluation. Clin Psychol Rev 8(1):77-100

46. Beck AT et al (1961) An inventory for measuring depression. Arch Gen Psychiatry 4(6):561-571

47. Buysse DJ et al (1989) The Pittsburgh Sleep Quality Index: a new instrument for psychiatric practice and research. Psychiatry Res 28(2):193-213

48. Rafiei M, Seifi A (2013) An investigation into the reliability and validity of beck anxiety inventory among the university students. JTBCP 7(27):37-46 
49. Vasegh S, Baradaran N (2014) Using the Persian-language version of the Beck Depression Inventory-II (BDI-II-Persian) for the screening of depression in students. J Nerv Ment Dis 202(10):738-744

50. Mesdaghinia E et al (2017) Clinical and metabolic response to selenium supplementation in pregnant women at risk for intrauterine growth restriction: randomized, double-blind, placebocontrolled trial. Biol Trace Elem Res 178(1):14-21

51. Rayman MP et al (2014) Effect of selenium on markers of risk of pre-eclampsia in UK pregnant women: a randomised, controlled pilot trial. Br J Nutr 112(1):99-111

52. Fordyce FM (2013) Selenium Deficiency and Toxicity in the Environment. In: Selinus O. (eds) Essentials of Medical Geology. Springer, Dordrecht. https://doi.org/10.1007/978-94-007-4375-5_ 16.

53. Nazemi L et al (2012) Selenium status in soil, water and essential crops of Iran. Iranian J Environ Health Sci Eng 9(1):11

54. Burk RF et al (2006) Effects of chemical form of selenium on plasma biomarkers in a high-dose human supplementation trial. Cancer Epidemiol Biomarkers Prev 15(4):804-810

55. Aldosary BM et al (2012) Case series of selenium toxicity from a nutritional supplement. Clin Toxicol (Phila) 50(1):57-64

56. Tara F et al (2010) Selenium supplementation and the incidence of preeclampsia in pregnant Iranian women: a randomized, doubleblind, placebo-controlled pilot trial. Taiwan J Obstet Gynecol 49(2):181-187

57. Rayman MP (2000) The importance of selenium to human health. Lancet 356(9225):233-241

58. Gómez $\mathrm{O}$ et al (2006) Sequential changes in uterine artery blood flow pattern between the first and second trimesters of gestation in relation to pregnancy outcome. Ultrasound Obstet Gynecol 28(6):802-808

59. Özkaya MO et al (2011) Effects of multivitamin/mineral supplementation on trace element levels in serum and follicular fluid of women undergoing in vitro fertilization (IVF). Biol Trace Elem Res 139(1):1-9

60. Ozkaya MO, Nazıroğlu M (2010) Multivitamin and mineral supplementation modulates oxidative stress and antioxidant vitamin levels in serum and follicular fluid of women undergoing in vitro fertilization. Fertil Steril 94(6):2465-2466

61. Kamali A, Amirani E, Asemi Z (2019) Effects of selenium supplementation on metabolic status in patients undergoing for coronary artery bypass grafting $(\mathrm{CABG})$ surgery: a randomized, double-blind, placebo-controlled trial. Biol Trace Elem Res 191(2):331-337

62. Yürekli VA, Nazıroğlu M (2013) Selenium and topiramate attenuates blood oxidative toxicity in patients with epilepsy: a clinical pilot study. Biol Trace Elem Res 152(2):180-186

63. Ju W et al (2017) The effect of selenium supplementation on coronary heart disease: a systematic review and meta-analysis of randomized controlled trials. J Trace Elem Med Biol 44:8-16

64. Savory LA (2012) et al., Selenium supplementation and exercise: effect on oxidant stress in overweight adults. Obesity (Silver Spring) 20(4):794-801
65. Köse SA, Nazıroğlu M (2014) Selenium reduces oxidative stress and calcium entry through TRPV1 channels in the neutrophils of patients with polycystic ovary syndrome. Biol Trace Elem Res 158(2):136-142

66. Ozturk IC et al (2008) Comparison of plasma malondialdehyde, glutathione, glutathione peroxidase, hydroxyproline and selenium levels in patients with vitiligo and healthy controls. Indian J Dermatol 53(3):106-110

67. Zeng J, Zhou J, Huang K (2009) Effect of selenium on pancreatic proinflammatory cytokines in streptozotocin-induced diabetic mice. J Nutr Biochem 20(7):530-536

68. He YT et al (2004) Therapeutic effects and molecular mechanisms of anti-fibrosis herbs and selenium on rats with hepatic fibrosis. World J Gastroenterol 10(5):703-706

69. Hawkes WC, Hornbostel L (1996) Effects of dietary selenium on mood in healthy men living in a metabolic research unit. Biol Psychiatry 39(2):121-128

70. Pittara T et al (2021) Pre-eclampsia and long-term health outcomes for mother and infant: an umbrella review. BJOG 128(9):1421-1430

71. Brussé I et al (2008) Impaired maternal cognitive functioning after pregnancies complicated by severe pre-eclampsia: a pilot case-control study. Acta Obstet Gynecol Scand 87(4):408-412

72. Thiagayson P et al (2013) Depression and anxiety in Singaporean high-risk pregnancies - prevalence and screening. Gen Hosp Psychiatry $35(2): 112-116$

73. Gruca-Stryjak K et al (2014) Is there a relationship between pregnancy induced hypertension and obstructive sleep apnea? Case report. Pneumonol Alergol Pol 82(2):156-162

74. Mokhber $\mathrm{N}$ et al (2011) Effect of supplementation with selenium on postpartum depression: a randomized double-blind placebocontrolled trial. J Matern Fetal Neonatal Med 24(1):104-108

75. Shor-Posner G et al (2003) Psychological burden in the era of HAART: impact of selenium therapy. Int J Psychiatry Med 33(1):55-69

76. Albuquerque RG, Tufik S, Andersen ML (2019) Benefits of selenium in the treatment of depression and sleep disorders. Sleep Breath 23(3):933-934

77. Tinggi U (2008) Selenium: its role as antioxidant in human health. Environ Health Prev Med 13(2):102-108

78 Solovyev $\mathrm{N}$ et al (2021) Selenium at the neural barriers: a review. Front Neurosci 15:630016

79 Naderi $\mathrm{M}$ et al (2021) A comprehensive review on the neuropathophysiology of selenium. Sci Total Environ 767:144329

80. Kohrle J et al (2005) Selenium, the thyroid, and the endocrine system. Endocr Rev 26(7):944-984

Publisher's Note Springer Nature remains neutral with regard to jurisdictional claims in published maps and institutional affiliations. 